\title{
Des réalités insoupçonnées
}

\section{Anna Sax}

Lic. oec. publ., MHA, membre de la rédaction

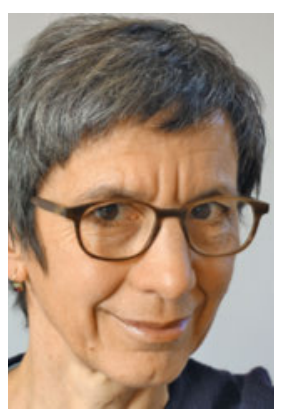

Je suis tombé récemment sur un livre dont la lecture m'a stupéfait et indigné: il y a des personnes qui sont tenues pour démentes et traitées en psychiatrie rien que parce qu'elles voient mal et que personne ne s'en est aperçu. Et lorsque leur problème finit par être identifié comme un handicap visuel, elles n'ont pas droit à des mesures d'intégration. Parce qu'elles sont âgées. À quoi bon vouloir encore intégrer des personnes âgées dépendantes et faibles de vue - et dans quoi, d'ailleurs? Elles peuvent s'estimer heureuses d'avoir un lit douillet, d'être nourries et d'avoir quelqu'un pour leur donner des soins et leur faire la lecture de temps en temps. N'ayant plus de tâches à accomplir, un homme ou une femme à la retraite n'a plus de fonction dans notre société. Une personne âgée dont la vue ou l'ouïe a baissé n'a qu'à s'y faire. Nombreux sont ceux, hélas, qui le voient ainsi, y compris parmi les premiers concernés. Bien qu'il soit de notoriété publique que la vue diminue en général avec l'âge, souvent ni la personne intéressée ni son entourage ne se rendent compte qu'elle ne voit plus comme avant. C'est dû en partie au fait que nous voyons "avec notre cerveau». Celui-ci peut par exemple compléter une image en cas de perte d'une partie du champ visuel ("filling-in») ou produire des hallucinations visuelles («syndrome de Charles Bonnet»). Résultat: une personne atteinte de cécité du champ visuel central pourra "voir» la table que lui fait miroiter le cerveau, mais non l'assiette posée dessus. Ou voir des obstacles où il n'y en a pas. Que faire alors? Que penser du grand-père qui se plaint d'être le seul à table à ne pas avoir reçu un verre de vin, alors que son verre se trouve devant lui? Ou de la belle-mère hantée par la peur de trébucher dans des trous qui n'existent pas? C'est évident: ces personnes sont démentes. Leurs proches le pensent, et elles finissent tôt ou tard par le croire elles-mêmes.

Mais il y a pire: même aux yeux des professionnels de la santé, les personnes âgées faibles de vue passent souvent pour démentes, comme le montre une étude présentée dans le livre et portant sur des patients atteints de cataracte. Il semble en effet que la perte d'acuité visuelle, si elle n'est pas diagnostiquée et traitée, mène à des pertes intellectuelles qui ressemblent à s'y méprendre à une démence. Or il s'avère ensuite que les personnes initialement évaluées comme démentes retrouvent leurs facultés cognitives après opération de la cataracte. Une autre étude aboutit au constat qu'un grand nombre de handicapé(e)s de la vue résidant dans des institutions de soins reçoivent une prise en charge focalisée sur la démence. Les éditrices concluent que la vraie fréquence de la démence pourrait être nettement réduite par une assistance à l'autonomie axée sur la réadaptation. La dépression, autre conséquence fréquente d'une perte visuelle, pourrait elle aussi être évitée moyennant un accompagnement et des soins adéquats. Il est sans doute inutile de mentionner que des phénomènes analogues sont observés chez les personnes souffrant de pertes auditives avec l'âge.

Le livre ouvre les yeux sur des réalités insoupçonnées. Même une personne qui perd la vue et l'ouïe avec l'âge peut mener une vie autonome et active si elle bénéficie d'un appui propice au renforcement de ses capacités. Les éditrices et autrices montrent les possibilités d'y parvenir, inspirées par de nombreux exemples de leur longue pratique. L'essence de l'ouvrage est un guide comportant 33 règles d'or sur la manière adéquate d'aborder une diminution de la vue chez une personne âgée. Cette histoire a aussi une dimension politique: le système d'assurances sociales ne prévoit pas de soutien approprié aux personnes qui développent un handicap à l'âge de la retraite. Leur période d'activité professionnelle étant révolue, l'intégration des personnes âgées n'est plus du ressort de l'AI, mais de l'assurancemaladie. Cette dernière paie les soins et la prise en charge médicale, mais ne verse rien pour les aider à reprendre activement leur vie en main. Pour ce qui est notamment des personnes âgées, il serait temps de prendre à cœur la tâche politique de leur intégration sociale et d'appuyer des mesures en ce sens, par exemple au moyen de prestations complémentaires à l'AVS. Par de telles aides, nous soutenons non seulement ces personnes, mais délestons aussi le système de santé et le système social. Car plus longtemps l'on pourra vivre sa vieillesse en toute autonomie, mieux l'on sera en mesure d'aider son prochain.

\section{Référence}

Fatima Heussler, Judith Wildi, et Magdalena Seibl (édit.), Menschen mit Sehbehinderung in Alterseinrichtungen. Gerontagogik und gerontagogische Pflege - Empfehlungen zur Inklusion, Seismo Verlag, Zurich 2016. 